\title{
Bilateral Extensive Emphysematous Pyelonephritis
}

\author{
A Case Report
}

\author{
Ing-Kit Lee ${ }^{a}$ Ching-Jung Hsieh ${ }^{b}$ Jien-Wei Liu ${ }^{a}$
}

Divisions of a Infectious Diseases and ${ }^{b}$ Endocrinology and Metabolism, Department of Internal Medicine, Chang Gung Memorial Hospital - Kaohsiung Medical Center, Chang Gung University College of Medicine, Taoyuan, Taiwan

\section{Key Words}

Bilateral emphysematous pyelonephritis · Hyperglycemic hyperosmolar state $\cdot$ Diabetes mellitus $\cdot$ Nephrostomy

\begin{abstract}
Objective: We report a case involving a patient with bilateral emphysematous pyelonephritis (EPN) who was successfully treated with antibiotics and nephrostomy sparing nephrectomy. Case Presentation and Intervention: A 54year-old diabetic man presented with the chief complaint of progressive abdominal pain for 10 days. Blood tests indicated acute renal failure and hyperglycemic hyperosmolar state. An abdominal radiograph disclosed gas distributed over regions where the bilateral kidneys are located and the right para-lumbosacral area. Computed tomography revealed bilateral enlarged kidneys with the presence of gas in the renal parenchyma and renal pelvis, left peri-renal space, bilateral ureter, bladder, prostate gland and right scrotum. Cultures of urine and blood grew Escherichia coli. The patient was successfully treated with antibiotics coupled with nephrostomy. Conclusion: Timely recognition and initiation of effective antibiotic therapy and nephrostomy may cure the patient with bilateral EPN, while sparing nephrectomy. Regarding clinical manifestations, EPN is nonspecific; hence, radiographs should be taken for diabetic patients with abdominal pain (especially those with poorly controlled blood sugar) to enable early diagnosis of potential EPN.
\end{abstract}

Copyright $\odot 2009$ S. Karger AG, Basel

\section{KARGER}

Fax +4161306 1234

E-Mail karger@karger.ch

www.karger.com (c) 2009 S. Karger AG, Basel

$1011-7571 / 09 / 0182-0149 \$ 26.00 / 0$

Accessible online at:

www.karger.com/mpp

\section{Introduction}

Emphysematous pyelonephritis (EPN) occurs particularly in patients with diabetes mellitus or obstructive uropathy $[1,2]$. EPN is rarely encountered in both kidneys concurrently. However, when EPN involves both kidneys, the affected patient is at a higher risk of developing renal failure and subject to higher mortality [2]. Effective therapeutic modalities for bilateral EPN are controversial, although bilateral nephrectomy is considered to be a life-saving measure $[1,2]$. We report a case involving a patient with bilateral EPN in which the gas produced extended into bilateral ureters, bladder, prostate gland and right scrotum, coupled with a hyperglycemic hyperosmolar state and acute renal failure.

\section{Case Report}

A 54-year-old man presented to our Emergency Service with progressive nonspecific abdominal pain of a 10-day duration. $\mathrm{He}$ had experienced malaise, polydipsia and weight loss 1 month earlier, and was not told that he had diabetes mellitus. On examination, he was consciously disoriented, but afebrile $\left(36.5^{\circ} \mathrm{C}\right)$, with a heart rate of 114 beats/min and a blood pressure of $126 / 63 \mathrm{~mm} \mathrm{Hg}$. His abdomen was distended with hypoactive bowel sounds. Clinical examination did not disclose signs or symptoms suggestive of peritonitis or a urinary tract infection.

The results of blood tests were: peripheral white blood cell count: $51.2 \times 10^{3} / \mathrm{mm}^{3}$ (normal, 3.9-10.6 $\times 10^{3} / \mathrm{mm}^{3}$ ) with $91 \%$ 


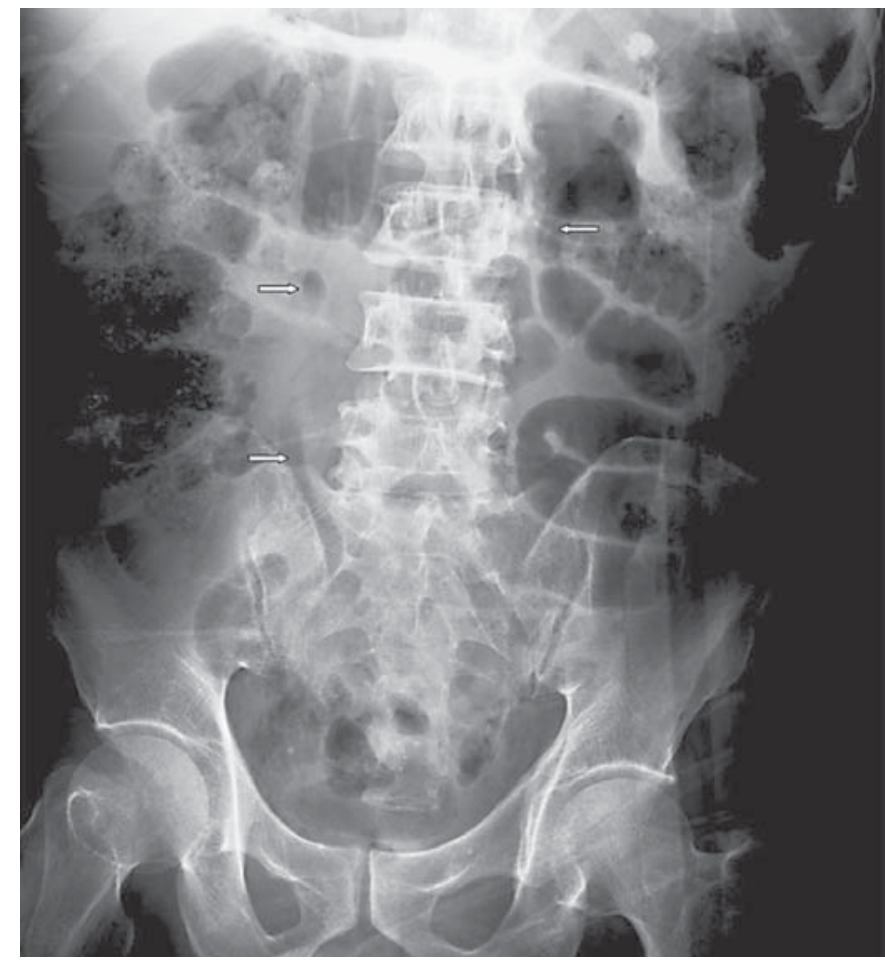

Fig. 1. Abdomen radiograph revealing unusual gas (arrows).

polymorphonuclear cells; platelet count: $300 \times 10^{3} / \mathrm{mm}^{3}$ (normal, $\left.150-400 \times 10^{3} / \mathrm{mm}^{3}\right)$; blood sugar: $618 \mathrm{mg} / \mathrm{dl}$ and osmolality: $356 \mathrm{mosm} / \mathrm{l}$ (normal, 275-295 mosm/l); creatinine: $8.3 \mathrm{mg} /$ dl (normal, 0.4-1.4 mg/dl); potassium: $6.5 \mathrm{mEq} / \mathrm{l}$ (normal, 3.0$4.8 \mathrm{mEq} / \mathrm{l}$ ); sodium: $115 \mathrm{mEq} / 1$ (normal, $134-148 \mathrm{mEq} / \mathrm{l}$ ), and glycosylated hemoglobin $15.7 \%$ (normal, 4.6-6.2\%). An abdomen radiograph showed gas distributed over regions where the bilateral kidneys are located and the right para-lumbosacral area (fig. 1). Urinalysis revealed hematuria and pyuria. Computed tomography (CT) showed bilateral enlarged kidneys with the presence of gas in the renal parenchyma and renal pelvis, left peri-renal space, bilateral ureter, bladder, prostate gland and right scrotum (fig. 2). No evidence of urinary tract obstruction was found.

Bilateral extensive EPN, hyperglycemic hyperosmolar state and acute renal failure were diagnosed. Intravenous fluid and insulin were given. Imipenem was intravenously administrated after sampling blood and urine for culture. Because of the patient's critical condition, anesthesis was considered too risky and nephrectomy was deferred. Hemodialysis was carried out for acute renal failure and hyperkalemia $5 \mathrm{~h}$ after his arrival, followed by CT-guided percutaneous nephrostomy for drainage of pus admixed with air from left kidney. The patient was then admitted for further conservative treatment; his general condition gradually improved $36 \mathrm{~h}$ after presentation to the hospital.

Cultures of urine, blood and pus all grew Escherichia coli. Antibiotic treatment was later switched to cefuroxime based on the results of antimicrobial susceptibility testing. The metabolic de- rangement was gradually corrected, and his renal function improved. The draining tube was removed from the left kidney on day 10 of hospitalization. The patient was released after 25 days of hospitalization.

\section{Discussion}

EPN is a life-threatening kidney infection, which particularly occurs in diabetics $[1,2]$. E. coli accounts for approximately $70 \%$ of all possible infectious etiologies in EPN [1-3]. Of note, the symptoms and signs for urinary tract infection in this reported case were not overt enough, which might result from the clinically distracting or masking effect by the overwhelming hyperglycemic hyperosmolar state [4], rendering negligence of the clinical presentations of urinary tract infection that would otherwise have been noticed. Our report underscores the importance of including EPN in the differential diagnosis of a diabetic patient presenting with abdominal pain regardless of the absence of symptoms/signs for urinary tract infection and/or fever. A delayed diagnosis may lead to rapid deterioration of EPN and development of septic shock, which carries a mortality rate of approximately $50 \%[1]$.

Stratified severity of EPN based on radiological manifestations and clinical outcomes was previously reported by Huang and Tseng [2] and Wan et al. [3]. EPN in our patient was extremely severe and involved both kidneys with dissemination of the gas generated by the pathogen in the urogenital system. Bilateral extensive EPN is rare, yet more life-threatening [2, 5-7]. Patients experiencing bilateral EPN with 2 or more indicators for profound sepsis (i.e., thrombocytopenia, acute renal failure, drowsiness and shock) are subject to significantly higher treatment failure rate when treated conservatively with nephrostomy drainage and antibiotic(s) [2]. With regards to our patient, the metabolic derangement in general and acute renal failure in particular raised the concern of whether or not he could tolerate an emergency bilateral nephrectomy, hence the decision was made to perform nephrostomy. Effective therapeutic modalities for bilateral EPN remain controversial [2, 5-7]. Although conservative treatment alone, type I EPN (renal parenchymal destruction with absence of fluid content or presence of streaky or mottled gas on CT, regardless of the existence of bubbly or loculated gas) [3], bilateral EPN and thrombocytopenia have each been identified as a significant risk factor for mortality in a meta-analysis [8], patients with bilateral EPN cured by medical treat- 

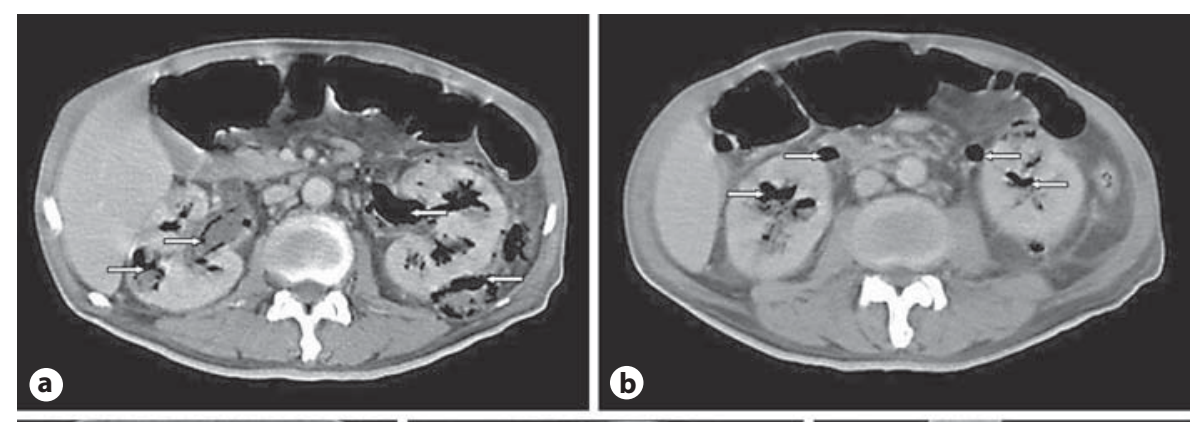

Fig. 2. Computed tomography showing bilateral enlarged kidney with presence of gas (arrows) in the parenchyma as well as within renal pelvis, left peri-renal space (a, b), bilateral ureter (b), bladder (c), prostate gland (d) and right scrotum (e).
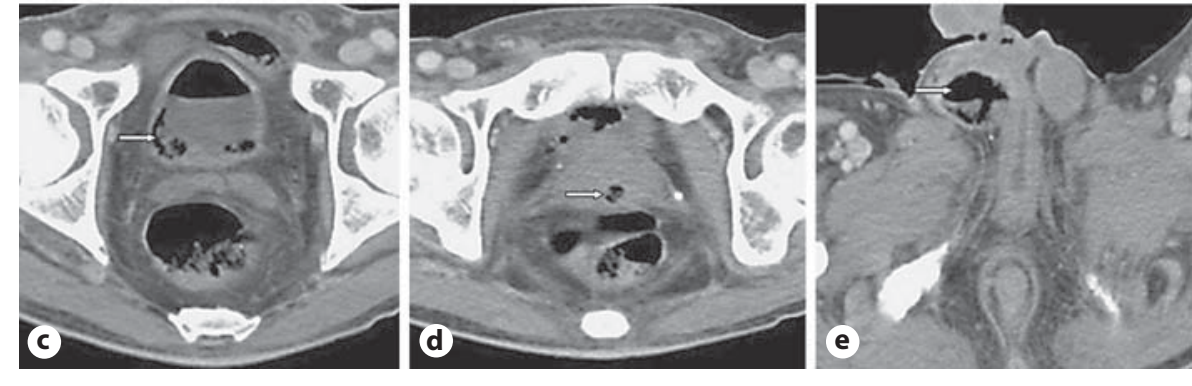

ment alone have been sporadically reported in the literature $[9,10]$.

Early percutaneous drainage could reduce bacterial burden, release the gas generated by the culprit pathogen and increase local antibiotic concentration in the inflammatory site leading to enhanced kidney perfusion. For our patient, the abdominal radiography taken at the Emergency Service played a key role in the successful treatment of his bilateral EPN because it disclosed clues to the presence of gas in the upper urinary tract, and thereby provided timely management guidance.

\section{Conclusion}

This case demonstrated that aggressive medical treatment with nephrostomy and antibiotics might be an effective alternative to surgical intervention even under such a critical condition, if conservative treatment is started early enough; nephrectomy and resultant permanent hemodialysis could therefore be avoided. This case also suggests that all diabetic patients with abdominal pain (regardless of the absence of fever) should have an abdominal radiograph taken.

\section{References}

$>1$ Chen MT, Huang CN, Chou YH, Huang CH, Chiang CP, Liu GC: Percutaneous drainage in the treatment of emphysematous pyelonephritis: 10-year experience. J Urol 1997;157: 1569-1573.

2 Huang JJ, Tseng CC: Emphysematous pyelonephritis: clinicoradiological classification, management, prognosis, and pathogenesis. Arch Intern Med 2000;160:797-805.

$\checkmark 3$ Wan YL, Lee TY, Bullard MJ, Tsai CC: Acute gas-producing bacterial renal infection: correlation between imaging findings and clinical outcome. Radiology 1996;198:433-438.
4 Dutta P, Bhansali A, Singh SK, Gupta KL, Bhat MH, Masoodi SR, Kumar Y: Presentation and outcome of emphysematous renal tract disease in patients with diabetes mellitus. Urol Int 2007;78:13-22.

$\checkmark 5$ Goyzueta JD, Katz R, Dumitrescu O, Choi HS, Kahn T: The disappearing kidney: a case of emphysematous pyelonephritis. Arch Intern Med 1994;154:2613-2615.

-6 McHugh TP, Albanna SE, Stewart NJ: Bilateral emphysematous pyelonephritis. Am J Emerg Med 1998;16:166-169.

7 Arun N, Hussain A, Kapoor MM, Abul F: Bilateral emphysematous pyelonephritis and emphysematous cystitis with autosomaldominant polycystic kidney disease: is conservative management justified? Med Princ Pract 2007;16:155-157.
Falagas ME, Alexiou VG, Giannopoulou KP, Siempos II: Risk factors for mortality in patients with emphysematous pyelonephritis: a meta-analysis. J Urol 2007;178:880-885.

-9 Tahir H, Thomas G, Sheerin N, Bettington H, Pattison JM, Goldsmith DJ: Successful medical treatment of acute bilateral emphysematous pyelonephritis. Am J Kidney Dis 2000;36:1267-1270.

10 Karasavidou L, Nikolaou S, Archontakis S, Papatheodorou G, Koroneos V, Drakoulis C: Nonsurgical treatment of bilateral emphysematous pyelonephritis in a diabetic patient. J Nephrol 2006;19:664-667. 